\title{
Exploration of the Forecast of Interbank Borrowing Rate Based on Artificial Intelligence
}

\author{
Ming $\mathrm{Lu}^{1}$, Ning $\mathrm{Yao}^{2}$, Xinxi $\mathrm{Lu}^{3}$, a) \\ ${ }^{1}$ Zhong Gang DaGong Technology, Beijing, China \\ 2. school of software, beihang university, Beijing, China \\ ${ }^{3}$ School of Electronic and Information Engineering, beihang university, Beijing, China \\ a) Corresponding Author Email: 30256663@qq.com
}

\begin{abstract}
In order to ensure the stability in financial markets and the position in international financial market game of China, this paper uses the method of artificial intelligence to research and forecast the interbank interest rates of banking industry, which is from the time dimension of the day wave. According to the characteristics of daily data, the appropriate algorithm was selected and the algorithm was further optimized. The results showed that the established prediction model had some scientific and applied value in the daily data forecast of overnight Shibor. Therefore, the establishment of the overnight Shibor decision system and the integration of the prediction model have a certain guiding effect on the participations of the money market.
\end{abstract}

Key words: Interbank rate, Artificial intelligence, BP neural network, Wavelet neural network.

\section{INTRODUCTION}

China's economic status has been continuously improved, and the importance of establishing and improving a strong financial market has become more and more prominent. Among them, gradual introduction of the marketoriented interest rate is the key of the reform of the financial field, which will directly affect the stability of China's financial markets and the position in international financial market game. The key of interest rate liberalization is to establish a benchmark interest rate. Benchmark interest rate refers to interest rate which has general reference meaning in the money market. Interbank offered rate is also called the interbank rates and is the interest rate which circulates the necessary funds between commercial banks in the monetary market, which is usually chosen as the benchmark interest rate in the world.

China's central bank takes Shibor as the benchmark interest rate in our country to develop (Peng Huafei, 2005), at present, Shibor offers eight varieties of interest rates, it is overnight $(\mathrm{O} / \mathrm{N})$, one week $(1 \mathrm{w})$, two weeks $(2 \mathrm{w})$, one month $(1 \mathrm{~m})$, two months $(2 \mathrm{~m})$, three months $(3 \mathrm{~m})$, six months $(6 \mathrm{~m})$, nine months $(9 \mathrm{~m})$ and one year $(1 \mathrm{y})$ respectively. Using the appropriate interest rate prediction method scientifically, Shibor prediction model is explored and established (Zhang Yaotian, 2007), the design of Shibor decision-making system is of great significance for the more effective financial regulation and more efficient money management of the banking industry.

\section{LITERATURE REVIEW}

The prediction research of the rate of inter-bank discharges is almost about the traditional financial time series model in the early stage (Wang Junsheng, 2010). For example, the ARIMA and GARCH models were established by Peng Huafei and Ren Zhaozhang in 2005 and they found that the ARIMA model had a good predictive ability in the prediction of the overnight rate of Chibor (Peng Huafei, 2005). In 2012, Xie Xiaolu forecasted Shibor of two weeks based on wavelet neural network and regressing-time series combination model, whose results showed that the error 
of the wavelet neural network was small, which has better fitting effect than the regressing-time series combination model (Xie Xiaolu, 2012). In 2015, Zhou Sicong compared and analyzed the volatility characteristics of Shibor and Libor, putting forward the forecasting model which combined the method of empirical mode decomposition (EMD) and Elman neural network (Zhou Sicong, 2015). Although the traditional financial time series method has a good effect on the forecast effect, there are some limitations. With the rapid development of artificial intelligence methods, especially the research work of artificial neural network, the neural network provides a new method for the modeling and solving high-dimensional nonlinear problems, which has been applied to financial data analysis, processing, and prediction gradually (Tian Min, 2009). On the basis of in-depth study of existing literature, this paper takes the Shibor as the research object, sthdies overnight Shibor prediction model based on artificial intelligence algorithm and the overnight Shibor daily fluctuations data. Overnight Shibor data from 2007 to 2015 were selected, almost covering all of the Shibor data released. So, it is more meaningful for the predicting effect and has more practical application value to establish prediction model in this time span.

\section{METHODOLOGY}

\section{Overnight Shibor Prediction Based on Artificial Intelligence}

The research object of this chapter is overnight Shibor daily data, research method is the artificial neural network, overnight Shibor daily data of January 4, 2007 to December 31, 2015 were selected for the empirical analysis of the forecasting model, covering all of the Shibor data released. Overnight Shibor is chosen as the research object, because in the interbank lending market at present, the trading volume of overnight varieties Shibor is largest, and accounts for a very large share, the research for the overnight Shibor has practical significance. Among 2248 overnight Shibor data, the first 2100 (January 1, 2007 - on May 29, 2015) data were intercepted as training data set, the remaining 148 (June 1, 2015 - December 31, 2015) data were chosen to be the test data. The following three models were used for the research:

BP neural network prediction model. In this paper, BP neural network prediction model is established firstly as a basic model, which can be used as the reference standard in further study.

Wavelet neural network prediction model. Wavelet neural network takes the topology structure of BP network as the theoretical basis and bases on the wavelet transform theory to construct feedforward neural network, on the one hand, it uses the advantages of neural network, that is, network self-learning and massively parallel computing, on the other hand, it fully combines the properties of wavelet transform localization, therefore, it has strong ability of fitting approximation and faster convergence speed.

Wavelet neural network prediction model optimized by cuckoo bird (CS). The prediction model of CS-wavelet neural network is established. In CS-wavelet neural network, each egg represents the initial weight and threshold of wavelet network, the iterative process is to constantly update location using CS algorithm and to optimize the initial weights and threshold, so as to meet the fitness functions until the optimum.

\section{Overnight Shibor Decision System}

In the practical application, the main participants in the money market include the people's bank of China, commercial Banks, enterprises and investors, etc. The participants have different strengths, positions and goals in the money market. Through the design of the system framework, we guide the relevant workers to make the prediction and judgment and make better decisions.

The central bank maintains the stable development of financial markets through opening market operations, formulating reserve requirements and other measures. With the gradual operation and maturity of Shibor, Shibor becomes one of the important tools for the economic environment regulation of the government. Commercial Banks, as the main players in the lending market, are naturally affected by the level of interest rates. Interbank workers can combine the condition of the bank's capital as well as the short-term and long-term goals and use the decision system to choose the appropriate time for the money lending considering varieties and the amount of money borrowed. The enterprises have different sensitivities to different operating activities, and Shibor decision-making system can be applied according to the enterprise situation. Individual investors are the important participants and vulnerable groups in the financial markets, countries shloud constantly strengthen the protection of individual investors from the policy, but the more important is the enhancement of the public investment philosophy. The higher the interest rate 
liberalization, the more obvious the investment level gap will be, and investors should learn to apply the Shibor decision system to guide their own investment behaviors.

\section{RESULTS AND DISCUSSION}

In the three models of this section, among 2248 overnight Shibor data, the first 2100 (January 1, 2007 - on May 29, 2015) data were intercepted as training data set, the remaining 148 (June 1, 2015 - December 31, 2015) data were chosen to be the test data. The average absolute error (MAE), mean square error (MSE) and mean percentage error (MAPE) were chosen as the indicators to measure the prediction error. The specific expressions are shown in formula (1), (2) and (3):

$$
\begin{gathered}
M A E=\frac{1}{n} \sum_{i=1}^{n}\left|X_{i}-\bar{X}_{i}\right| \\
M S E=\frac{1}{n} \sum_{i=1}^{n}\left(X_{i}-\bar{X}_{i}\right)^{2} \\
M A P E=\frac{1}{n} \sum_{i=1}^{n}\left|\frac{X_{i}-\bar{X}_{i}}{X_{i}}\right| \times 100 \%
\end{gathered}
$$

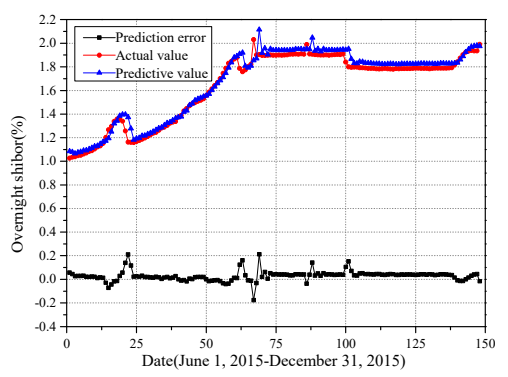

FIGURE 1. Prediction effect diagram - BP neural network model

Figure 1 showed the forecasting effect of BP neural network model. It could be seen from figure 1 that in the BP neural network, the stable stage of the first 60 data in the time series prediction of overnight Shiobr better fitted the loan interest rate volatility, while the fluctuation was large and the fitting performance was normal. When the deviation was predicted, there was a large deviation in the fitting of the latter nearly 90 data points. On the whole, BP neural network prediction model basically fitted the fluctuation trend of the discharges rate, but the error was still relatively large. Among them, the value of MAPE was 2.3978, the value of MAE was 0.0388 , and the MSE value was 0.0028 .

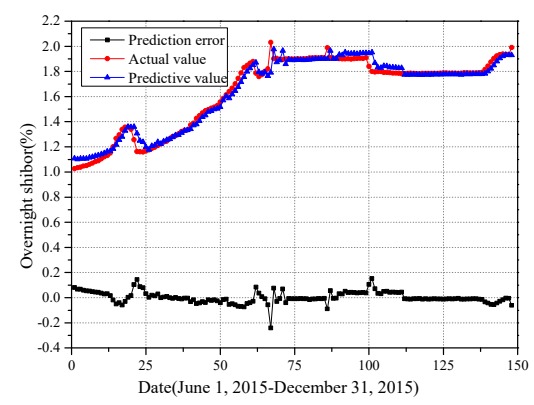

FIGURE 2. Prediction effect diagram - wavelet neural network model 
Figure 2 shows the forecasting effect of wavelet neural network model, from which it could be seen that in the wavelet neural network, the fitting effect of the first 25 data and the 25 data between 50 and 75 was not very good and the prediction error was big. In the forecast of the remaining 100 data points, except for the data points with individual extreme fluctuations, the prediction accuracy was very high, and the relative error was very small, which was basically consistent with the daily real value data of real discharges rate. Among them, the value of MAPE was 1.8689, the value of MAE was 0.0281, and the MSE value was 0.0017 .

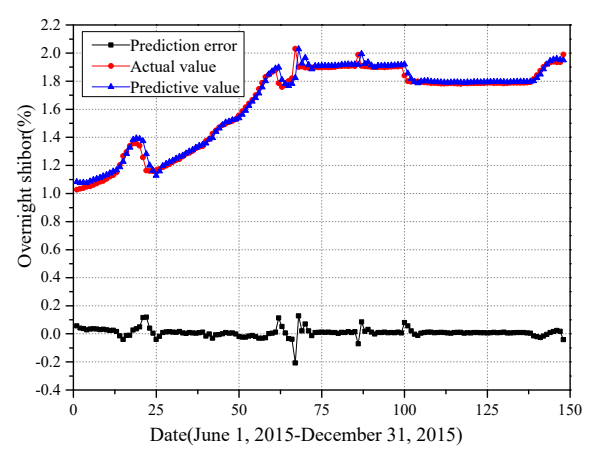

FIGURE 3. Prediction effect diagram - CS-wavelet neural network model

Figure 3 shows the predicting effect of CS-wavelet neural network model. It can be seen from the figure 3 that compared to the wavelet neural network, the first 25 data in CS-wavelet neural network model had a better fitting effect in the forecast and the 25 dates between 50-75 in CS-wavelet neural network model also had a better fitting effect than wavelet neural network model, the capturing of the extreme point and fitting ability was stronger, the deviation and hysteresis appeared less, and the ability of predicting was further improved. The model basically fitted the daily data of the interest rate. Among them, the value of MAPE was 1.4072, MAE's value was 0.0216, and MSE value was 0.0012 .

Overnight Shibor prediction model was based on artificial intelligence, we used BP neural network, wavelet neural network and CS-wavelet neural network to predict the overnight Shibor daily data and paid close attention to the daily fluctuations of loan interest rate. For interbank interest rates which had such high-dimensional nonlinear time series data, the overall predicting effect of the BP neural network prediction model was general, in the process of fitting for the test set, once the volatile inflection point appeared, the prediction effect of test samples after turning point would be greatly affected, reducing the whole prediction precision. The prediction effect of wavelet neural network in the loan interest rate volatility was quite good, then the use of the cuckoo search algorithm for parameter optimization of the wavelet neural network had markedly improved the prediction accuracy of the predicting model, the optimized model showed good effect in the daily data prediction of overnight Shibor, which could use the predictive value to assist decision-making. Overnight Shibor prediction errors of the BP neural network, wavelet neural network, and CSwavelet neural network based on artificial intelligence algorithm were shown in table 1 :

TABLE 1. Comparison of model prediction errors

\begin{tabular}{|c|c|c|c|}
\hline & \multicolumn{3}{|c|}{ Prediction model } \\
\hline Evaluation index & BP neural network & $\begin{array}{c}\text { Wavelet neural } \\
\text { network }\end{array}$ & $\begin{array}{c}\text { CS-wavelet neural } \\
\text { network }\end{array}$ \\
\hline MAPE & 2.3978 & 1.8689 & 1.4072 \\
\hline MAE & 0.0388 & 0.0281 & 0.0216 \\
\hline MES & 0.0028 & 0.0017 & 0.0012 \\
\hline
\end{tabular}




\section{CONCLUSION}

For interbank interest rates which have such high-dimensional nonlinear time series data, the overall predicting effect of the BP neural network prediction model is general, in the process of fitting for the test set, once the volatile inflection point appeares, the prediction effect of test samples after turning point will be greatly affected. The prediction effect of wavelet neural network in the loan interest rate volatility is quite good, then the use of the cuckoo search algorithm for parameter optimization of the wavelet neural network had markedly improves the prediction accuracy of the pre-dicting model, the optimized model shows good effect in the daily data prediction of overnight Shibor, which can use the predictive value to assist decision-making. In the short-term interest rate daily data forecast, the forecast model has very good performance and can be used to predict the volatility

\section{ACKNOWLEDGEMENTS}

Avoid the stilted expression, “One of us (R. B. G.) thanks...” Instead, try "R. B. G. thanks". Do NOT put sponsor acknowledgements in the unnumbered footnote on the first page, but at here.

\section{REFERENCES}

1. Huafei, P. (2005). Study on the forecast model of inter-bank lending rate in China. Southern Financial, (4), 2325.

2. Yaotian, Z. (2007). Application of ARIMA model in the forecast of interbank borrowing rate. Journal of Taiyuan Normal University: Natural Science Edition, 2(1), 22-33.

3. Junsheng, W. (2010). Application of the Elman neural network in interbank loan rate analysis. Statistics \& Information Forum, 25(1), 84-87.

4. Xiaolu, X. (2012). Study on Shibor prediction based on wavelet neural network. Financial theory and practice, (8):57-60.

5. Xiaowei, S. (2014). Research on the application of combinatorial model based on artificial intelligence optimization in the prediction of interbank lending rate. Lanzhou University.

6. Shicong, Z. (2015). Analysis and forecast of the fluctuation characteristics of interbank lending rate in Shanghai. Beyond the Cultural Arbitrary. Jinan University.

7. Min, T. (2009). Shanghai interbank offered rate (Shi bor) based on time series. Journal of Ningxia University: Humanities and Social Sciences Edition, 31(2), 129-132. 